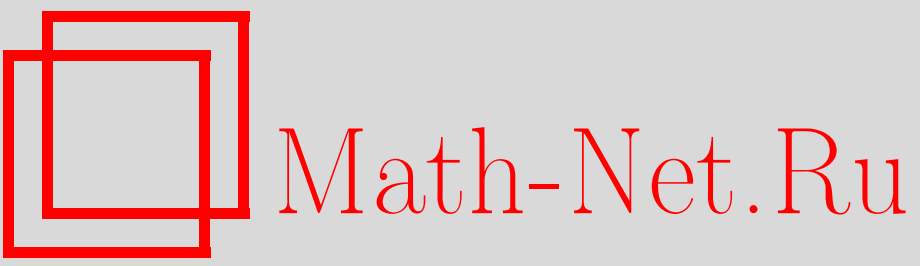

О. И. Завьялов, А. М. Малокостов, Квантовая теория поля с нефоковскими асимптотическими полями: существование $S$-матрицы, ТMФ, 1999, том 121, номер 1, 25-39

DOI: https://doi.org/10.4213/tmf796

Использование Общероссийского математического портала Math-Net.Ru подразумевает, что вы прочитали и согласны с пользовательским соглашением

http: //www . mathnet.ru/rus/agreement

Параметры загрузки:

IP : 54.205 .225 .156

26 апреля 2023 г., 13:51:03 
ТЕОРЕТИЧЕСКАЯ

И МАТЕМАТИЧЕСКАЯ

ФИЗИКА

Том 121, № 1

октябрь, 1999

(C) 1999 г. $\quad$ О.И. Завьялов*, А.М. Малокостов*

\section{КВАНТОВАЯ ТЕОРИЯ ПОЛЯ С НЕФОКОВСКИМИ АСИМПТОТИЧЕСКИМИ ПОЛЯМИ: СУЩЕСТВОВАНИЕ $S$-МАТРИЦЫ}

Построено семейство релятивистски-инвариантных производящих функционалов для нефоковских представлений канонических перестановочных соотношений вида

$$
F\left(f^{*}, g\right)=\exp \left\{\gamma \int \frac{d \mathbf{k}}{\omega(\mathbf{k})} f^{*}(\mathbf{k}) g(\mathbf{k})\right\}
$$

Проанализирован первый порядок по константе связи соответствующей квантовой теории поля, имеющей в качестве in-асимптотики свободное поле $\varphi(x)$, отвечающее такому функционалу. В первом порядке доказана унитарная эквивалентность свободных асимптотических out- и in-полей, и тем самым доказано существование унитарной матрицы рассеяния. Одновременно доказано, что кинематика "нефооковской квантовой теории поля" существенно богаче стандартной, а именно, показано, что в отличие от стандартного случая $S$-матрица не совпадает с обычной хронологически упорядоченной экспонентой от лагранжиана взаимодействия.

\section{1. ВВЕДЕНИЕ}

Стандартная схема квантовой теории поля состоит в следующем. Ищется операторнозначное решение квантовых уравнений движения со специальными асимптотическими условиями: при больших отрицательных значениях времени $t$ квантовое гейзенбергово поле $\Phi(x)$ должно приближаться к свободному in-полю $\varphi_{\text {in }}(x)$ :

$$
\Phi(t, \mathbf{x}) \approx \varphi_{\mathrm{in}}(x)
$$

при $t \rightarrow-\infty$. Если сушествует также свободное асимптотическое out-поле $\varphi_{\text {out }}(x)$, т.е. при больших временах гейзенбергово поле становится сколь угодно близким к $\varphi_{\text {out }}(x)$, и если in- и out-поля унитарно-эквивалентны, то можно определить $S$-матрицу:

$$
\varphi_{\text {out }}(x)=S^{+} \varphi_{\text {in }}(x) S
$$

* Математический институт им. В. А. Стеклова РАН, Москва, Россия 
Обычно используется фоковское представление для іп-поля. Однако оно имеет ряд недостатков. В частности, оно не допускает никакой разумной координатной интерпретации. Например, любое состояние, которое естественно интерпретировать как локализованную частицу, распространяется со скоростью $v>c$. Можно было бы попытаться преодолеть указанную трудность, используя подход Вигнера [1]. А именно, в квантовой механике строится так называемая функция Вигнера, которая в некоторых случаях дает альтернативный способ физической интерпретации формальной математической схемы в терминах распределения квазивероятностей в фазовом пространстве исходной системы. К сожалению, эти квазивероятности не обязательно положительны. При этом патологические случаи с нарушением релятивистской причинности возникают именно для отрицательных квазивероятностей.

Первая трудность, которая возникает на этом пути, связана с тем, что до недавнего времени не было разумного обобщения функции Вигнера на релятивистский случай. В работе [2] мы привели такое обобщение. Однако эта функция также не является положительно-определенной в обычном квантовомеханическом случае. Более того она обладает неудовлетворительными трансформационными свойствами по отношению к группе Лоренца. Очевидно, что обе эти особенности, вообше говоря, зависят от выбора конкретного представления поля $\varphi_{\text {in }}$. Поэтому мы надеемся, что нам удастся найти такое представление, в котором функция Вигнера “более нормальна" и поэтому координатная интерпретация квантовой теории релятивистских частиц более разумна.

Отметим сразу, что до настоящего времени нам не удалось найти такое представление, поэтому наша идея остается пока только программой действий. Тем не менее уже сейчас ясно, что в некотором смысле теория с нефоковскими асимптотическими полями имеет более богатую структуру, чем обыгная “фоковская” теория. Поэтому представляется полезным рассмотреть типичную модель с каким-либо конкретно выбранным нефоковским представлением асимптотических полей независимо от того, как выглядят функция Вигнера и проблема координатной интерпретации этой теории в целом.

В ранних работах $[3,4]$ было предложено использовать нефоковские бесконечные тензорные произведения представлений для полей $\varphi_{\text {in }}(x)$ для изучения влияния такого выбора на общую структуру квантовой теории поля. К сожалению, эти работыне получили продолжения в связи с отсутствием тогда известных бесконечных тензорных произведений представлений, удовлетворяющих необходимым основным принципам (например, лоренш-инвариантности). Настояшая работа продолжает это обсуждение, используя недавно найденное бесконечно приводимое нефоковское лоренц-инвариантное представление поля $\Phi(x)$. Отметим, что для построения этого нового представления операторов рождения и уничтожения $a^{+}(\mathbf{k})$ и $a(\mathbf{k})$ (которые входят в свободное нефоковское поле $\left.\varphi_{\text {in }}(x)\right)$ достаточно, как обычно, построить так называемый производяший функционал $F\left(f^{*}, g\right)$, где $f$ и $g$-гладкие пробные функции. Здесь

$$
\varphi_{\mathrm{in}}(x) \equiv \varphi(x)=\frac{1}{\sqrt{2(2 \pi)^{3}}} \int \frac{d \mathbf{k}}{\omega(\mathbf{k})}\left[a^{+}(\mathbf{k}) e^{i \omega(\mathbf{k}) t-i \mathbf{k} \mathbf{x}}+a(\mathbf{k}) e^{-i \omega(\mathbf{k}) t+i \mathbf{k x}}\right],
$$

а операторы $a^{+}$и $a$ подчиняются стандартным коммутационным соотношениям

$$
\left[a(\mathbf{k}), a^{+}(\mathbf{p})\right]=\omega(\mathbf{k}) \delta(\mathbf{k}-\mathbf{p}) .
$$


Производящий функционал $F$ должен удовлетворять специальным соотношениям положительной определенности. Представление в соответствующем гильбертовом пространстве может быть получено из этого функционала посредством дифференцирования по $f^{*}$ и $g$ на основании теоремы реконструкции [3]. Например,

$$
\left\langle 0\left|a^{+}(\mathbf{k}) a(\mathbf{p})\right| 0\right\rangle=\left.\omega(\mathbf{k}) \omega(\mathbf{p}) \frac{\delta}{\delta f^{*}(\mathbf{k})} \frac{\delta}{\delta g(\mathbf{p})} F\left(f^{*}, g\right)\right|_{f^{*}=g=0} .
$$

В терминах теоремы реконструкции производящий функционал имеет следуюший смысл:

$$
\begin{aligned}
F\left(f^{*}, g\right) & =\left\langle 0\left|\exp \left\{f^{*} \circ a^{+}\right\} \exp \{g \circ a\}\right| 0\right\rangle \equiv \\
& \equiv\left\langle 0\left|\exp \left\{\int f^{*}(\mathbf{k}) a^{+}(\mathbf{k}) \frac{d \mathbf{k}}{\omega(\mathbf{k})}\right\} \exp \left\{\int g(\mathbf{p}) a(\mathbf{p}) \frac{d \mathbf{p}}{\omega(\mathbf{p})}\right\}\right| 0\right\rangle,
\end{aligned}
$$

где $|0\rangle$ - соответствуюший циклический вектор данного представления.

Чтобы определить представление $U(\Lambda, r)$ группы Пуанкаре, положим

$$
U(\Lambda, r)|0\rangle=|0\rangle
$$

На других векторах гильбертова пространства преобразование $U(\Lambda, r)$ определено соотношением

$$
U a^{\#}(\mathbf{k})|0\rangle=U a^{\#}(\mathbf{k}) U^{+} U|0\rangle=a^{\#}(\mathbf{\Lambda} \mathbf{k}) e^{ \pm i k r}|0\rangle .
$$

Отсюда следует, что полевые операторы преобразуются обычным образом:

$$
U a^{\#}(\mathbf{k}) U^{+}=a^{\#}(\mathbf{\Lambda} \mathbf{k}) e^{ \pm i k r}
$$

Здесь $a^{\#}$ обозначает либо $a^{+}$, либо $a$; знаки + и - в экспонентах соответствуют случаям операторов $a^{+}$и $a$.

Упомянутые вьше условия положительности обеспечивают положительность скалярного произведения в соответствуюшем гильбертовом пространстве. Например, одно из таких условий дается соотношением

$$
\sum_{i, j} \bar{z}_{i} z_{j} F\left(f_{i}^{*}, f_{j}\right) \geqslant 0
$$

для любого конечного набора ненулевых комплексных чисел $z_{1}, z_{2}, \ldots$ и для любого набора пробных функций $f_{i}$. Это соотношение символизирует положительность нормы вектора

$$
\sum_{j} z_{j} \exp \left\{\int \frac{d \mathbf{k}}{\omega(\mathbf{k})} f_{j}(\mathbf{k}) a(\mathbf{k})\right\}|0\rangle .
$$

Другие аналогичные соотношения можно легко получить при другом выборе вектора, определяюшего правую часть равенств типа (4). (Напомним, что производящий функционал, соответствуюший фоковскому представлению, где циклический вектор подчинен дополнительному условию $a(\mathbf{k})|0\rangle=0$ для любых $\mathbf{k}$, тождественно равен единице: $F\left(f^{*}, g\right) \equiv 1 ;$ при этом циклический вектор назьвается вакуумом.) 
Предположим, что у нас имеются два производящих функционала $F_{1}\left(f^{*}, g\right)$ и $F_{2}\left(f^{*}, g\right)$. Эти функционалы определяют два набора канонических операторов $a_{1}, a_{1}^{+}$ и $a_{2}, a_{2}^{+}$. Легко проверить, что, если $F_{1} \equiv F_{2}$, то два этих набора унитарно-эквивалентны, и что унитарный оператор $V$, осушествляюший эту эквивалентность, может быть явно построен. Именно

$$
V\left|0_{1}\right\rangle=\left|0_{2}\right\rangle, \quad V P\left(a_{1}^{+}, a_{1}\right)\left|0_{1}\right\rangle=P\left(a_{2}^{+}, a_{2}\right)\left|0_{2}\right\rangle,
$$

где $P$ - произвольно упорядоченный полином по $a^{+}$и $a$. Если отождествить соответствуюшие гильбертовы пространства $H_{1}$ и $H_{2}$ (т.е. положить $\left|0_{1}\right\rangle=\left|0_{2}\right\rangle=|0\rangle$ ), получится так называемое условие стабильности циклического вектора

$$
V|0\rangle=|0\rangle
$$

Отметим, что если $F_{1} \neq F_{2}$, это не означает, что эти два представления обязательно неэквивалентны. Например, если выбрать $\left|0_{2}\right\rangle \neq\left|0_{1}\right\rangle$ в том же самом неприводимом гилбертовом пространстве $H_{1}$, то производяший функционал изменится, но унитарная эквивалентность сохранится.

Наконец, скажем несколько слов о (не)приводимости представления и о том, как это связано со свойствами производяшего функционала. Если производящий функционал $F\left(f^{*}, g\right)$ может быть представлен в виде суммы

$$
F\left(f^{*}, g\right)=c_{1} F_{1}\left(f^{*}, g\right)+c_{2} F_{2}\left(f^{*}, g\right)
$$

где $c_{1}$ и $c_{2}$ - положительные числа, а $F_{1}$ и $F_{2}$ не совпадают тождественно с $F$ и удовлетворяют необходимым условиям, налагаемым на производящий функционал, то представление, определяемое исходным функционалом $F$, будет приводимым и может быть разложено в прямую сумму представлений, определяемых каждым из функционалов $F_{1}$ и $F_{2}$ по отдельности.

\section{2. ПРОИЗВОДЯщИЙ ФУНКЦИОНАЛ}

Теперь выберем модельньй производяший функционал для рассматриваемого нефоковского представления. Предположим, что этот функционал $F\left(f^{*}, g\right)$ зависит только от комбинации

$$
X=\int \frac{d \mathbf{k}}{\omega(\mathbf{k})} f^{*}(\mathbf{k}) g(\mathbf{k}),
$$

т.е. от лоренц-инвариантного скалярного произведения функций $f$ и $g$. Таким образом, комбинация $X$ будет пуанкаре-инвариантной.

Такой выбор может показаться довольно произвольным. Однако он обеспечивает стабильность циклического вектора по отношению к преобразованиям из группы Пуанкаре, что, как отмечалось, представляется крайне желательным. 
Вообше говоря, пуанкаре-инвариантность соответствующего представления будет обеспечена, если производящий функционал $F\left(f^{*}, g\right)$ будет зависеть также от норм каждой из функций $f$ и $g$ в отдельности, т.е. от комбинаций

$$
Y=\int \frac{d \mathbf{k}}{\omega(\mathbf{k})} f^{*}(\mathbf{k}) f(\mathbf{k}), \quad Z=\int \frac{d \mathbf{k}}{\omega(\mathbf{k})} g^{*}(\mathbf{k}) g(\mathbf{k}) .
$$

Однако мы будем рассматривать только случай зависимости от $X$, поскольку структура квантовой теории поля (в рамках теории возмушений) может быть легко обобшена на эти два случая.

Сформулируем теперь некоторые технические ограничения, которые мы собираемся наложить на производящий функционал. Мы хотим, чтобы у нас сохранилась возможность перехода на дискретную решетку в импульсном пространстве, т.е. использования трехмерной решетки вместо непрерывного пространства $\mathbb{R}^{3}$. В этом случае нам необходимо сделать замену

$$
\begin{gathered}
\int \frac{d \mathbf{p}}{\omega(\mathbf{p})} f^{*}(\mathbf{p}) a^{+}(\mathbf{p}) \longrightarrow \sum_{i} \frac{1}{\omega\left(\mathbf{p}_{i}\right)} f^{*}\left(\mathbf{p}_{i}\right) a^{+}\left(\mathbf{p}_{i}\right), \\
\int \frac{d \mathbf{k}}{\omega(\mathbf{k})} g(\mathbf{k}) a(\mathbf{k}) \longrightarrow \sum_{i} \frac{1}{\omega\left(\mathbf{p}_{i}\right)} g\left(\mathbf{p}_{i}\right) a\left(\mathbf{p}_{i}\right) .
\end{gathered}
$$

Нетривиальные “дискретные” коммутационные соотношения примут вид

$$
\left[a\left(\mathbf{k}_{i}\right), a^{+}\left(\mathbf{k}_{j}\right)\right]=\omega\left(\mathbf{k}_{i}\right) \delta_{i, j} .
$$

Здесь предполагается, что последовательности $\left\{f\left(\mathbf{p}_{j}\right)\right\}$ и $\left\{g\left(\mathbf{p}_{j}\right)\right\}$ состоят из конечного числа ненулевых элементов. Мы опять потребуем, чтобы производящий функционал зависел только от комбинации

$$
X=\sum_{i} \frac{1}{\omega\left(\mathbf{k}_{i}\right)} f^{*}\left(\mathbf{k}_{i}\right) g\left(\mathbf{k}_{i}\right)
$$

Известно (см., например, [3]), что с точностью до унитарной эквивалентности бесконечные тензорные произведения определяются поведением циклического вектора данного бесконечного тензорного произведения при больших номерах $j$, а выбор компонент при малых $j$ несуществен. Поэтому мы хотим использовать “одни и те же” циклические векторы для каждой степени свободы $j$. В этом случае производящий функционал будет произведением производящих функционалов для каждого номера $j$ :

$$
F\left(f^{*}, g\right)=\prod_{j} F\left(x_{j}\right),
$$

где $x_{j}=f_{j}^{*} g_{j}$. Естественное обобшение этого требования состоит в следуюшем. Предположим, что набор номеров $j$ с ненулевыми $f\left(\mathbf{k}_{j}\right)$ и $g\left(\mathbf{k}_{j}\right)$ состоит из двух непересекающихся групп $K_{1}$ и $K_{2}$ таких, что комбинация $X$ из $(7)$ принимает форму

$$
X=X_{1}+X_{2}
$$


где

$$
X_{1}=\sum_{i \in K_{1}} \frac{1}{\omega\left(\mathbf{k}_{i}\right)} f^{*}\left(\mathbf{k}_{i}\right) g\left(\mathbf{k}_{i}\right), \quad X_{2}=\sum_{i \in K_{2}} \frac{1}{\omega\left(\mathbf{k}_{i}\right)} f^{*}\left(\mathbf{k}_{i}\right) g\left(\mathbf{k}_{i}\right) .
$$

В этой ситуации мы опять хотим, чтобы

$$
F(X)=F\left(X_{1}\right) F\left(X_{2}\right)
$$

Уравнение (10) совместимо с ограничением, чтобы функционал $F$ зависел лиш от комбинации $X$, только если

$$
F(X)=\exp \{\gamma X\}
$$

Предположим, что именно этот функционал соответствует нашему базовому представлению для свободного in-поля $\varphi_{\text {in }}(x)$. Априори мы не можем утверждать, что при таком выборе будут выполнены упомянутые вьше условия положительности. Мы не будем проверять явно эти условия. Вместо этого в следуюшем разделе мы опишем явно конкретное представление операторов рождения и уничтожения $a^{+}(\mathbf{k}), a(\mathbf{k})$, соответствуюшее производящему функционалу вида (11). Это представление окажется бесконечно приводимым. В частности, помимо основного поля $\varphi(x)$, в пространстве представления будет действовать еще и другое поле $\psi(x)$, коммутирующее с $\varphi(x)$. Эта дополнительная степень свободы собственно и позволит, как мы увидим, построить приемлемую матрицу рассеяния.

Сейчас мы проверим явно условия положительности функционала (11) для случая одной степени свободы (например, $j=1, \mathbf{k}_{1}=0$ и соответственно $\omega\left(\mathbf{k}_{1}\right)=m$ ). При этом

$$
X=x_{1}=f_{1}^{*} g_{1} \equiv x \equiv f^{*} g
$$

(мы положили массу равной единице: $m=1$ ).

Построим другие производящие функционалы $F_{n}(x)$, соответствующие другому выбору нормированных циклических векторов $\left|0_{n}\right\rangle$ (в том же гильбертовом пространстве):

$$
\left|0_{n}\right\rangle=\frac{\left(a^{+}\right)^{n}}{\sqrt{n !}}|0\rangle
$$

где $|0\rangle$ - обычный вакуум, который обращается в нуль при действии оператора $a$. (Очевидно, что в силу теоремы фон Неймана [5] для случая одной степени свободы все такие циклические векторы приводят к разным производящим функционалам, но все соответствуюшие представления унитарно-эквивалентны фоковскому представлению канонических коммутационных соотношений.) Найдем $F_{n}$. Получим

$$
F_{n}\left(f^{*}, g\right)=\frac{1}{n !}\left\langle 0\left|a^{n} e^{f^{*} a^{+}} e^{g a}\left(a^{+}\right)^{n}\right| 0\right\rangle .
$$

Легко проверить, что

$$
F_{n}\left(f^{*}, g\right)=L_{n}(-x),
$$


где $x=f^{*} g$, а $L_{n}-$ полиномы Лагерра:

$$
L_{n}(-x)=\frac{1}{n !} e^{-x} \frac{d^{n}}{d x^{n}}\left(x^{n} e^{x}\right) .
$$

Очевидно, что по построению все функции $F_{n}(x)$ удовлетворяют необходимым условиям положительности. С другой стороны, известна формула [6]

$$
e^{\gamma x}=\sum_{n=0}^{\infty} \frac{\gamma^{n}}{(1+\gamma)^{n+1}} L_{n}(-x) .
$$

Таким образом, видно, что функционал $e^{\gamma x}$ при $\gamma \geqslant 0$ равен бесконечной сумме (с положительными коэффициентами) функционалов $F_{n}(x)$, удовлетворяющих необходимым условиям положительности. Это означает, что и сам исходный функционал $F(x)=e^{\gamma x}$ также удовлетворяет этим условиям. Кроме того, это означает, что представление, соответствуюшее функционалу $F(x)=e^{\gamma x}$, бесконечно приводимо. Соответственно наше базовое представление (если оно сушествует), отвечающее функционалу $F(X)=e^{\gamma X}$ также будет приводимым. В следуюшем разделе мы явно проверим, что этот функционал действительно положительно-определен.

\section{3. СВОБОДНОЕ ПОЛЕ $\varphi(x)$}

Вернемся к непрерывному случаю и явно построим представление асимптотического свободного поля $\varphi(x)$. Для этой цели рассмотрим две пары операторов рождения и уничтожения $b^{+}(\mathbf{k}), c^{+}(\mathbf{k}), b(\mathbf{k}), c(\mathbf{k})$ в обычном фоковском представлении канонических коммутационных соотношений с обшим вакуумным вектором $|0\rangle$, т.е. для всех $\mathbf{k}$,

$$
b(\mathbf{k})|0\rangle=0, \quad c(\mathbf{k})|0\rangle=0 .
$$

Рассмотрим линейные комбинации (при любом $\gamma>0$ )

$$
a^{+}(\mathbf{k})=\sqrt{1+\gamma} b^{+}(\mathbf{k})+\sqrt{\gamma} c(\mathbf{k}), \quad a(\mathbf{k})=\sqrt{1+\gamma} b(\mathbf{k})+\sqrt{\gamma} c^{+}(\mathbf{k}) .
$$

Легко проверить, что операторы $a^{+}(\mathbf{k}), a(\mathbf{p})$ подчиняются каноническим коммутационным соотношениям

$$
\left[a(\mathbf{k}), a^{+}(\mathbf{p})\right]=\omega(\mathbf{k}) \delta(\mathbf{k}-\mathbf{p}) .
$$

С другой стороны, предположим, что вакуумный вектор $|0\rangle$ - нормированный циклический вектор, и вычислим производящий функционал $F\left(f^{*}, g\right)$ :

$$
F\left(f^{*}, g\right)=\left\langle 0\left|\exp \left\{f^{*} \circ a^{+}\right\} \exp \{g \circ a\}\right| 0\right\rangle .
$$

Можно легко убедиться, что этот функционал в точности совпадает с $F(X)$ :

$$
F\left(f^{*}, g\right)=\left\langle 0\left|\exp \left\{f^{*} \circ a^{+}\right\} \exp \{g \circ a\}\right| 0\right\rangle=\exp (\gamma X) .
$$

Это автоматически решает проблему всех условий положительности. 
Используя $a^{+}(\mathbf{k})$ и $a(\mathbf{k})$ в качестве операторов рождения и уничтожения, построим свободное поле $\varphi(x)$ :

$$
\varphi(x)=\sqrt{1+\gamma} \chi(x)+\sqrt{\gamma} \eta(x),
$$

где $\chi(x)$ - обычное квантовое свободное поле,

$$
\chi(x)=\frac{1}{\sqrt{2(2 \pi)^{3}}} \int \frac{d \mathbf{p}}{\omega(\mathbf{p})}\left[b^{+}(\mathbf{p}) e^{i \omega(\mathbf{p}) x_{0}-i \mathbf{p} \mathbf{x}}+b(\mathbf{p}) e^{-i \omega(\mathbf{p}) x_{0}+i \mathbf{p x}}\right],
$$

а $\eta(x)$, так сказать, “антиквантовое" свободное поле,

$$
\eta(x)=\frac{1}{\sqrt{2(2 \pi)^{3}}} \int \frac{d \mathbf{p}}{\omega(\mathbf{p})}\left[c^{+}(\mathbf{p}) e^{-i \omega(\mathbf{p}) x_{0}+i \mathbf{p} \mathbf{x}}+c(\mathbf{p}) e^{i \omega(\mathbf{p}) x_{0}-i \mathbf{p} \mathbf{x}}\right] .
$$

Когда мы говорим, что $\eta(x)$ - антиквантовое поле, мы имеем в виду, что поле $\eta(x)$ удовлетворяет "неправильным" коммутационным соотношениям

$$
[\dot{\eta}(t, \mathbf{x}), \eta(t, \mathbf{y})]=i \delta(\mathbf{x}-\mathbf{y})
$$

а поле $\chi(x)$ подчиняется "нормальным" соотношениям

$$
[\dot{\chi}(t, \mathbf{x}), \chi(t, \mathbf{y})]=-i \delta(\mathbf{x}-\mathbf{y}) .
$$

Отметим, что, как уже было сказано, при преобразовании из группы Пуанкаре $(\Lambda, a)$ операторы $a(\mathbf{k}), a^{+}(\mathbf{p})$ преобразуются стандартным образом:

$$
\begin{gathered}
U(\Lambda, a) a^{+}(\mathbf{k}) U^{+}(\Lambda, a)=e^{i k a} a^{+}(\mathbf{\Lambda} \mathbf{k}), \\
U(\Lambda, a) a(\mathbf{k}) U^{+}(\Lambda, a)=e^{-i k a} a(\boldsymbol{\Lambda} \mathbf{k}),
\end{gathered}
$$

а $c$ и $c^{+}$имеют "аномальные" трансформационные свойства:

$$
\begin{aligned}
U(\Lambda, a) c^{+}(\mathbf{k}) U^{+}(\Lambda, a) & =e^{-i k a} c^{+}(\mathbf{\Lambda} \mathbf{k}), \\
U(\Lambda, a) c(\mathbf{k}) U^{+}(\Lambda, a) & =e^{i k a} c(\mathbf{\Lambda} \mathbf{k}) .
\end{aligned}
$$

Заметим, что поле $\eta(x)$ может рассматриваться как обычное свободное поле с обратным знаком аргумента $x$.

Сделаем теперь еще одно важное замечание. Кроме операторов $a(\mathbf{k})$ и $a^{+}(\mathbf{k})$, сушествует другой канонический набор операторов $d(\mathbf{k})$ и $d^{+}(\mathbf{k})$, коммутирующих с операторами $a$ и $a^{+}$:

$$
d(\mathbf{k})=\sqrt{1+\gamma} c(\mathbf{k})+\sqrt{\gamma} b^{+}(\mathbf{k}), \quad d^{+}(\mathbf{k})=\sqrt{1+\gamma} c^{+}(\mathbf{k})+\sqrt{\gamma} b(\mathbf{k}) .
$$

Соответственно возникает новое поле $\psi(x)$ :

$$
\psi(x)=\frac{1}{\sqrt{2(2 \pi)^{3}}} \int \frac{d(\mathbf{k})}{\omega(\mathbf{k})}\left[d^{+}(\mathbf{k}) e^{i \omega(\mathbf{k}) t-i \mathbf{k x}}+d(\mathbf{k}) e^{-i \omega(\mathbf{k}) t+i \mathbf{k} \mathbf{x}}\right],
$$

которое коммутирует с полем $\varphi(x)$. 


\section{4. ВЗАИМОДЕЙСТВУЮЩЕЕ ПОЛЕ. МОДЕЛЬ $\lambda \Phi^{4}$}

Обозначим взаимодействуюшее “гейзенбергово" поле через $\Phi(x)$ (это аналог обычного вайтмановского поля в обычном фоковском случае). Наша конструкция теории возмушений для этого поля будет базироваться на подходе Боголюбова [7] (наши построения будут близки к версии боголюбовского подхода, описанной в первой главе книги [8]). Главная величина в этом подходе - так называемый ток $J(x)$, задаваемый потенциалом $\mathfrak{S}(\varphi)$ вне массовой поверхности (см. [8]). Ток $J(x)$ является, так сказать, "градиентом" потенциала $\mathfrak{S}$. Точнее, имеется следующая связь между потенциалом и током:

$$
J(x)=\frac{1}{i} \mathfrak{S}^{+} \otimes \frac{\delta \mathfrak{S}}{\delta \varphi(x)},
$$

где каждый символ может быть редуцирован на массовую поверхность, а $\otimes$ означает произведение соответствуюших операторов на массовой поверхности. В обычном фоковском случае функционал $\mathfrak{S}(\varphi)$ после редукции на массовую поверхность дает $S$-матрицу, так что потенциал $\mathfrak{S}(\varphi)$ и $S$-матрица вне массовой поверхности совпадают. В настоящей работе мы попытаемся действовать по аналогии со стандартной процедурой. Для начала мы проверим согласованность процедуры в первом порядке теории возмушений. Мы покажем, что в нашем случае потенциал $\mathfrak{S}(\varphi)$ и $S$-матрица $S(\varphi)$ не совпадают. Будет также использован лагранжиан

$$
L=\frac{\lambda}{4} \varphi^{4}
$$

(что бы он ни означал) как лагранжиан нашей теории и положено (в первом порядке по константе связи $\lambda$ )

$$
\mathfrak{S}(\varphi)=1+i \int d^{4} x \frac{\lambda}{4}: \varphi^{4}:
$$

где нормальное произведение : : означает виковское упорядочение по отношению к операторам $b$ и $c$. Ясно, что в первом порядке по $\lambda$ ток $J(x)$ должен равняться $\lambda: \varphi^{3}(x):$ с любым упорядочением, которое обеспечивает корректность этого оператора. Очевидно, что упорядочение : : удовлетворяет этому условию, так что потенциал $\mathfrak{S}$ определяет хорошо определенную замену для величины $\Phi^{3}(x)$ в первом порядке теории возмушений.

Таким образом, нелинейная часть квантовых уравнений движения хорошо определена (по крайней мере, в первом порядке), и можно определить взаимодействуюшее поле $\Phi(x)$ посредством соотношения Янга-Фельдмана

$$
\Phi(x)=\varphi(x)+\int d y D^{\mathrm{ret}}(x-y) J(y),
$$

где $D^{\text {ret }}$ - запаздывающая функция:

$$
\begin{aligned}
D^{\text {ret }}(x) & =\frac{1}{(2 \pi)^{4}} \int d^{4} k e^{-i k x} \frac{1}{m^{2}-k^{2}+i k_{0} \cdot 0}= \\
& =\theta\left(x_{0}\right)\left(D^{-}(x)-D^{-}(-x)\right)=\theta\left(x_{0}\right) D(x),
\end{aligned}
$$

2 Теоретическая и математическая физика, т. 121, № 1, 1999 г. 
а $D(x)$ - перестановочная функция Паули-Иордана. Очевидно, что $\Phi(x)$ удовлетворяет уравнению типа

$$
\left(\square+m^{2}\right) \Phi(x)=\lambda: \Phi^{3}(x)
$$

т.е. подходящему квантовому уравнению движению. Отметим, что в пределе $t=x_{0} \rightarrow$ $-\infty$ интегральный член в уравнении (16) обрашается в нуль, и мы получаем

$$
\Phi(x) \approx \varphi(x)
$$

Другими словами, асимптотическое іп-поле совпадает с $\varphi(x)$ :

$$
\varphi_{\mathrm{in}}(x)=\varphi(x)
$$

и поэтому $\Phi(x)$ есть решение корректно сформулированной квантовой задачи.

$\mathrm{C}$ другой стороны, в пределе больших положительных времен $t=x_{0} \rightarrow+\infty \theta$-функция в (17) может быть заменена единицей, так что получаем

$$
\Phi(x) \approx \varphi_{\text {out }}(x)
$$

где

$$
\varphi_{\text {out }}(x)=\varphi_{\text {in }}(x)+\int d y D(x-y) J(y) .
$$

Соотношения (19) и (20) позволяют определить величины на массовой поверхности, a именно in- и out-операторы рождения и уничтожения:

$$
a_{\mathrm{in}}(\mathbf{k})=a(\mathbf{k}), \quad a_{\mathrm{in}}^{+}(\mathbf{k})=a^{+}(\mathbf{k})
$$

и

$$
a_{\text {out }}(\mathbf{k})=a(\mathbf{k})+\delta a(\mathbf{k}), \quad a_{\text {out }}^{+}(\mathbf{k})=a^{+}(\mathbf{k})+\delta a^{+}(\mathbf{k}) .
$$

Можно убедиться, что с точностью до старших порядков

$$
\begin{aligned}
\delta a(\mathbf{k})= & i \lambda \frac{3}{4(2 \pi)^{2}} \int \frac{d \mathbf{q}_{1}}{\omega\left(\mathbf{q}_{1}\right)} \frac{d \mathbf{q}_{2}}{\omega\left(\mathbf{q}_{2}\right)} \frac{d \mathbf{q}_{3}}{\omega\left(\mathbf{q}_{3}\right)} \delta\left(\omega(\mathbf{k})-\omega\left(\mathbf{q}_{1}\right)-\omega\left(\mathbf{q}_{2}\right)+\omega\left(\mathbf{q}_{3}\right)\right) \times \\
& \times \delta\left(\mathbf{k}-\mathbf{q}_{1}-\mathbf{q}_{2}+\mathbf{q}_{3}\right) \vdots a\left(\mathbf{q}_{1}\right) a\left(\mathbf{q}_{2}\right) a^{+}\left(\mathbf{q}_{3}\right) \vdots \\
\delta a^{+}(\mathbf{k})= & -i \lambda \frac{3}{4(2 \pi)^{2}} \int \frac{d \mathbf{q}_{1}}{\omega\left(\mathbf{q}_{1}\right)} \frac{d \mathbf{q}_{2}}{\omega\left(\mathbf{q}_{2}\right)} \frac{d \mathbf{q}_{3}}{\omega\left(\mathbf{q}_{3}\right)} \delta\left(\omega(\mathbf{k})+\omega\left(\mathbf{q}_{1}\right)-\omega\left(\mathbf{q}_{2}\right)-\omega\left(\mathbf{q}_{3}\right)\right) \times \\
& \times \delta\left(\mathbf{k}+\mathbf{q}_{1}-\mathbf{q}_{2}-\mathbf{q}_{3}\right) \vdots a\left(\mathbf{q}_{1}\right) a^{+}\left(\mathbf{q}_{2}\right) a^{+}\left(\mathbf{q}_{3}\right) \vdots .
\end{aligned}
$$




\section{5. МАТРИЦА РАССЕЯНИЯ}

Теперь найдем, как изменяется производящий функционал при переходе от in-операторов $a, a^{+}$к оut-операторам $a+\delta a, a^{+}+\delta a^{+}$. В результате простых, но громоздких вычислений (см. приложение) получаем, что

$$
F^{\text {out }}\left(f^{*}, g\right)=\left\langle 0\left|\exp \left\{f^{*} \circ a^{+}+f^{*} \circ \delta a^{+}\right\} \exp \{g \circ a+g \circ \delta a\}\right| 0\right\rangle=F\left(f^{*}, g\right)+O\left(\lambda^{2}\right) .
$$

Таким образом, в нашем приближении производящий функционал не изменяется. Как известно, это означает, что out-операторы и in-операторы унитарно-эквивалентны. Тем самым мы получаем, что матрица рассеяния существует, по крайнем мере, в первом порядке по константе $\lambda$. Этот результат оказался для нас неожиданным. В самом деле, в обычном фоковском подходе сушествует прозрачный механизм сохранения производящего функционала. В первом порядке теории фозмущений этот механизм работает благодаря условиям $a|0\rangle=0$ и $\langle 0| a^{+}=0$. Этот механизм позволяет нам также отождествить потенциал $\mathfrak{S}$ и матрицу рассеяния $S(\varphi)$ вне массовой поверхности. В нашем случае такое отождествление невозможно. В самом деле, мы знаем, что $S|0\rangle=|0\rangle$. С другой стороны, $\mathfrak{S}|0\rangle \neq|0\rangle$. Кажется, что налицо противоречие. Разрешение парадокса сушественно использует дополнительные степени свободы в нашем іn-пространстве $H_{1}$. Напомним, что существует второе поле $\psi(x)$, коммутируюшее с полем $\varphi(x)$. Очевидно, что "потенциал" для тока

$$
J(x)=\frac{1}{i} \frac{\delta \mathfrak{S}}{\delta \varphi(x)}
$$

определен только с точностью до членов, не зависяших от $\varphi$. В нашем случае сушествуют нетривиальные члены этого типа. Именно можно выбрать (в первом порядке)

$$
S(\varphi)=1+i \int d^{4} x \frac{\lambda}{4}: \varphi^{4}:-i \int d^{4} x \frac{\lambda}{4}: \psi^{4}:
$$

Легко увидеть, что исходньй “ток” (в первом порядке) можно переписать в виде

$$
J(x)=\frac{1}{i} \frac{\delta S}{\delta \varphi(x)}
$$

Кроме того, можно проверить, что при таком выборе

$$
S(\varphi)|0\rangle=|0\rangle
$$

$$
S \otimes a(\mathbf{k}) \otimes S^{+}=a_{\text {out }}(\mathbf{k}), \quad S \otimes a^{+}(\mathbf{k}) \otimes S^{+}=a_{\text {out }}^{+}(\mathbf{k}) .
$$

Тем самым получаем, что $S(\varphi)$ можно интерпретировать как $S$-матрицу. 


\section{6. ОБСУЖДЕНИЕ}

В заключение заметим, что квантовая теория поля с нефоковскими асимптотическими полями, по-видимому, существует, но обладает некоторыми особенностями. Главная из них состоит в том, что обычное соотношение

$$
S(\varphi)=T \exp \left(i \int L(x) d x\right)
$$

где $L$ - лагранжиан, в таких теориях может нарушаться.

Что же касается высших порядков теории возмущений, то пока мы можем очень мало сказать о них. Может случиться, что существование таких теорий будет запрещено какими-то ограничениями, проявляюшимися в старших порядках. В любом случае пока вопрос остается открытым, и мы собираемся обсудить высшие порядки в следуюших публикациях.

\section{ПРИЛОЖЕНИЕ}

\section{Вычисление производящего функционала в первом порядке теории возмущений}

Здесь мы найдем производящий функционал в первом порядке по $\lambda$. Имеем

$$
\begin{aligned}
F^{\text {out }}\left(f^{*}, g\right) & =\left\langle 0\left|\exp \left\{f^{*} \circ a^{+}+f^{*} \circ \delta a^{+}\right\} \exp \{g \circ a+g \circ \delta a\}\right| 0\right\rangle= \\
& =F\left(f^{*}, g\right)+\delta F\left(f^{*}, g\right)+O\left(\lambda^{2}\right) .
\end{aligned}
$$

Разлагая экспоненты в ряд, находим, что $\delta F$ дается формулой

$$
\begin{aligned}
\delta F\left(f^{*}, g\right)= & \left\langle 0\left|\left(\bar{f} \circ \delta a^{+}\right) \exp \{g \circ a\}\right| 0\right\rangle+ \\
& +\left\langle 0\left|\exp \left\{\bar{f} \circ a^{+}\right\}(g \circ \delta a)\right| 0\right\rangle+ \\
& +\left\langle 0\left|\exp \left\{\bar{f} \circ a^{+}\right\} \sum_{l=2}^{\infty} \frac{1}{l !} \sum_{k=0}^{l-1}(g \circ a)^{k}(g \circ \delta a)(g \circ a)^{l-k-1}\right| 0\right\rangle+ \\
& +\left\langle 0\left|\sum_{n=2}^{\infty} \frac{1}{n !} \sum_{m=0}^{n-1}\left(\bar{f} \circ a^{+}\right)^{m}\left(\bar{f} \circ \delta a^{+}\right)\left(\bar{f} \circ a^{+}\right)^{n-m-1} \exp \{g \circ a\}\right| 0\right\rangle \equiv \\
\equiv & \delta_{1} F+\delta_{2} F+\delta_{3} F+\delta_{4} F .
\end{aligned}
$$

В дальнейшем мы будем использовать следующие важные тождества: если $\left[a(\mathbf{k}), a^{+}\left(\mathbf{k}^{\prime}\right)\right]=\omega(\mathbf{k}) \delta\left(\mathbf{k}-\mathbf{k}^{\prime}\right)$, то

$$
\begin{aligned}
e^{\bar{u} \circ a} a^{+}(\mathbf{k}) e^{-\bar{u} \circ a} & =a^{+}(\mathbf{k})+\bar{u}(\mathbf{k}), \\
e^{-u \circ a^{+}} a(\mathbf{k}) e^{u \circ a^{+}} & =a(\mathbf{k})+u(\mathbf{k})
\end{aligned}
$$

для любых (как фоковских, так и нефоковских) операторов $a, a^{+}$. 
Напомним, что

$$
a=\sqrt{1+\gamma} b+\sqrt{\gamma} c^{+}, \quad a^{+}=\sqrt{1+\gamma} b^{+}+\sqrt{\gamma} c
$$

Таким образом, для первого слагаемого в (25) имеем

$$
\begin{aligned}
\delta_{1} F & \equiv\left\langle 0\left|\left(\bar{f} \circ \delta a^{+}\right) \exp \{g \circ a\}\right| 0\right\rangle=\left\langle 0\left|\left(\bar{f} \circ \delta a^{+}\right) e^{\sqrt{\gamma} g \circ c^{+}}\right| 0\right\rangle= \\
& =\left\langle 0\left|e^{\sqrt{\gamma} g \circ c^{+}} e^{-\sqrt{\gamma} g \circ c^{+}}\left(\bar{f} \circ \delta a^{+}\right) e^{\sqrt{\gamma} g \circ c^{+}}\right| 0\right\rangle= \\
& =\left\langle 0\left|e^{-\sqrt{\gamma} g \circ c^{+}}\left(\bar{f} \circ \delta a^{+}\right) e^{\sqrt{\gamma} g \circ c^{+}}\right| 0\right\rangle= \\
& =\left\langle 0\left|\bar{f} \circ \widetilde{\delta a^{+}}\right| 0\right\rangle,
\end{aligned}
$$

где тильда над $\delta a^{+}$означает, что мы должны сдвинуть все операторы $c(\mathbf{k})$, входяшие в $\delta a^{+}: c(\mathbf{k}) \rightarrow c(\mathbf{k})+\sqrt{\gamma} g(\mathbf{k})$. Использовано также очевидное равенство $\langle 0| e^{\sqrt{\gamma} g \circ c^{+}}=$ $\langle 0|$. Принимая во внимание выражение $(24)$, получаем, что $\delta_{1} F \equiv 0$. Аналогично можно показать, что второе слагаемое, обозначенное в $(25)$ через $\delta_{2} F$, также равно нулю.

Рассмотрим теперь член $\delta_{3} F$. Имеем

$$
\begin{aligned}
\delta_{3} F & \equiv\left\langle 0\left|e^{\bar{f} \circ a^{+}} \sum_{l=2}^{\infty} \frac{1}{l !} \sum_{k=0}^{l-1}(g \circ a)^{k}(g \circ \delta a)(g \circ a)^{l-k-1}\right| 0\right\rangle= \\
& =\left\langle 0\left|e^{\sqrt{\gamma} \bar{f} \circ c^{+}} \sum_{l=2}^{\infty} \frac{1}{l !} \sum_{k=0}^{l-1}(g \circ a)^{k}(g \circ \delta a)(g \circ a)^{l-k-1}\right| 0\right\rangle= \\
& =\left\langle 0\left|e^{\sqrt{\gamma} \bar{f} \circ c^{+}} \sum_{l=2}^{\infty} \frac{1}{l !} \sum_{k=0}^{l-1}(g \circ a)^{k}(g \circ \delta a)(g \circ a)^{l-k-1} e^{-\sqrt{\gamma} \bar{f} \circ c^{+}} e^{\sqrt{\gamma} \bar{f} \circ c^{+}}\right| 0\right\rangle= \\
& =\left\langle 0\left|e^{\sqrt{\gamma} \bar{f} \circ c^{+}} \sum_{l=2}^{\infty} \frac{1}{l !} \sum_{k=0}^{l-1}(g \circ a)^{k}(g \circ \delta a)(g \circ a)^{l-k-1} e^{-\sqrt{\gamma} \bar{f} \circ c^{+}}\right| 0\right\rangle= \\
& =\left\langle 0\left|\sum_{l=2}^{\infty} \frac{1}{l !} \sum_{k=0}^{l-1}[g \circ(\sqrt{1+\gamma} b+\gamma \bar{f})]^{k}(g \circ \widetilde{\delta a})\left[g \circ\left(\sqrt{\gamma} c^{+}+\gamma \bar{f}\right)\right]^{l-k-1}\right| 0\right\rangle
\end{aligned}
$$

(здесь мы использовали то, что $\langle 0| c^{+}=0$ и $b|0\rangle=0$ ).

Обозначим

$$
d \mu \equiv \frac{3}{4(2 \pi)^{2}} \frac{d \mathbf{q}_{1}}{\omega\left(\mathbf{q}_{1}\right)} \frac{d \mathbf{q}_{2}}{\omega\left(\mathbf{q}_{2}\right)} \frac{d \mathbf{q}_{3}}{\omega\left(\mathbf{q}_{3}\right)} \delta\left(\omega(\mathbf{k})+\omega\left(\mathbf{q}_{1}\right)-\omega\left(\mathbf{q}_{2}\right)-\omega\left(\mathbf{q}_{3}\right)\right) \delta\left(\mathbf{k}+\mathbf{q}_{1}-\mathbf{q}_{2}-\mathbf{q}_{3}\right)
$$


Используя это обозначание, можно переписать $\delta_{3} F$ следуюшим образом:

$$
\begin{aligned}
\delta_{3} F= & i \lambda \int d \mu g(\mathbf{k}) \sum_{l=2}^{\infty} \frac{1}{l !} \sum_{k=0}^{l-1}\langle 0|[\sqrt{1+\gamma}(g \circ b)+\gamma(\bar{f} \circ g)]^{k} \times \\
& \times \gamma^{2} \bar{f}\left(\mathbf{q}_{1}\right) \bar{f}\left(\mathbf{q}_{2}\right)\left(\sqrt{1+\gamma} b^{+}\left(\mathbf{q}_{3}\right)+\sqrt{\gamma} c\left(\mathbf{q}_{3}\right)\right) \times \\
& \times\left[\sqrt{\gamma}\left(g \circ c^{+}\right)+\gamma(\bar{f} \circ g)\right]^{l-k-1}|0\rangle= \\
= & i \lambda \gamma^{2} \int d \mu g(\mathbf{k}) \sum_{l=2}^{\infty} \frac{1}{l !} \sum_{k=0}^{l-1}\left\{\sqrt{1+\gamma}\langle 0|[\sqrt{1+\gamma}(g \circ b)+\gamma(\bar{f} \circ g)]^{k} \times\right. \\
& \times \bar{f}\left(\mathbf{q}_{1}\right) \bar{f}\left(\mathbf{q}_{2}\right) b^{+}\left(\mathbf{q}_{3}\right)\left[\sqrt{\gamma}\left(g \circ c^{+}\right)+\gamma(\bar{f} \circ g)\right]^{l-k-1}|0\rangle+ \\
& \left.+\sqrt{\gamma}\langle 0|[\sqrt{1+\gamma}(g \circ b)+\gamma(\bar{f} \circ g)]^{k} \bar{f}\left(\mathbf{q}_{1}\right) \bar{f}\left(\mathbf{q}_{2}\right) c\left(\mathbf{q}_{3}\right)\right) \times \\
& \left.\times\left[\sqrt{\gamma}\left(g \circ c^{+}\right)+\gamma(\bar{f} \circ g)\right]^{l-k-1}|0\rangle\right\}= \\
= & i \lambda \gamma^{2} \int d \mu g(\mathbf{k}) \sum_{l=2}^{\infty} \frac{1}{l !} \sum_{k=0}^{l-1}\left\{\sqrt{1+\gamma} \bar{f}\left(\mathbf{q}_{1}\right) \bar{f}\left(\mathbf{q}_{2}\right)[\gamma(\bar{f} \circ g)]^{l-k-1} \times\right. \\
& \times\left\langle 0\left|[\sqrt{1+\gamma}(g \circ b)+\gamma(\bar{f} \circ g)]^{k} b^{+}\left(\mathbf{q}_{3}\right)\right| 0\right\rangle+ \\
& +\sqrt{\gamma}[\gamma(\bar{f} \circ g)]^{k} \bar{f}\left(\mathbf{q}_{1}\right) \bar{f}\left(\mathbf{q}_{2}\right) \times \\
& \left.\times\left\langle 0\left|c\left(\mathbf{q}_{3}\right)\left[\sqrt{\gamma}\left(g \circ c^{+}\right)+\gamma(\bar{f} \circ g)\right]^{l-k-1}\right| 0\right\rangle\right\} .
\end{aligned}
$$

Чтобы вычислить эту величину, разложим выражения в квадратных скобках по формуле бинома Ньютона:

$$
[\sqrt{1+\gamma}(g \circ b)+\gamma(\bar{f} \circ g)]^{k}=\sum_{m=0}^{k} \frac{k !}{m !(k-m) !}[\sqrt{1+\gamma}(g \circ b)]^{m}[\gamma(\bar{f} \circ g)]^{k-m} .
$$

Отлично от нуля только слагаемое при $m=1$ (поскольку мы имеем лишь один оператор $b^{+}$). Аналогично для второго слагаемого в фигурных скобках в (28) получаем

$$
\left[\sqrt{\gamma}\left(g \circ c^{+}\right)+\gamma(\bar{f} \circ g)\right]^{l-k-1}=\sum_{m=0}^{l-k-1} \frac{(l-k-1) !}{m !(l-k-1-m) !}\left[\sqrt{\gamma}\left(g \circ c^{+}\right)\right]^{m}[\gamma(\bar{f} \circ g)]^{l-k-1-m} .
$$

Опять-таки единственный ненулевой член в этой сумме - при $m=1$.

В конце концов, опуская простые промежуточные выкладки, получаем

$$
\begin{aligned}
\delta_{3} F & =i \lambda \gamma^{2} \int d \mu g(\mathbf{k}) g\left(\mathbf{q}_{3}\right) \bar{f}\left(\mathbf{q}_{1}\right) \bar{f}\left(\mathbf{q}_{2}\right) \frac{1+2 \gamma}{2} \sum_{l=2}^{\infty} \frac{1}{l !} l(l-1)[\gamma(\bar{f} \circ g)]^{l-2}= \\
& =i \lambda \gamma^{2} \frac{1+2 \gamma}{2} e^{\gamma(\bar{f} \circ g)} \int d \mu g(\mathbf{k}) g\left(\mathbf{q}_{3}\right) \bar{f}\left(\mathbf{q}_{1}\right) \bar{f}\left(\mathbf{q}_{2}\right)
\end{aligned}
$$


или

$$
\begin{aligned}
\delta_{3} F= & i \lambda \gamma^{2} \frac{1+2 \gamma}{2} \frac{3}{4(2 \pi)^{2}} e^{\gamma(\bar{f} \circ g)} \int \frac{d \mathbf{q}_{1}}{\omega\left(\mathbf{q}_{1}\right)} \frac{d \mathbf{q}_{2}}{\omega\left(\mathbf{q}_{2}\right)} \frac{d \mathbf{q}_{3}}{\omega\left(\mathbf{q}_{3}\right)} \times \\
& \times \delta\left(\omega(\mathbf{k})+\omega\left(\mathbf{q}_{1}\right)-\omega\left(\mathbf{q}_{2}\right)-\omega\left(\mathbf{q}_{3}\right)\right) \delta\left(\mathbf{k}+\mathbf{q}_{1}-\mathbf{q}_{2}-\mathbf{q}_{3}\right) \times \\
& \times g(\mathbf{k}) g\left(\mathbf{q}_{3}\right) \bar{f}\left(\mathbf{q}_{1}\right) \bar{f}\left(\mathbf{q}_{2}\right) .
\end{aligned}
$$

Последнее слагаемое в $(25)$ (обозначенное $\delta_{4} F$ ) вычисляется точно так же, и в результате имеем

$$
\delta_{4} F=-\delta_{3} F
$$

Соответственно все поправки первого порядка по константе связи сокращаются, и мы получаем

$$
\delta F=\delta_{3} F+\delta_{4} F=0 .
$$

Благодарности. Работа выполнена при поддержке Российского фонда фундаментальных исследований, гранты № 99-01-00033 и № 99-01-00119D.

\section{Список литературы}

[1] E. P. Wigner. Phys. Rev. 1932. V. 40. P. 749.

[2] О. И. Завьялов, А. М. Малокостов. ТМФ. 1999. Т. 119. № 1. С. 67.

[3] О.И. Завьялов, В. Н. Сушко. Неэквивалентные представления соотношений коммутации в физике бесконечных систем. В сб.: Статистическая физика и квантовая теория поля. Посвящен памяти С. В. Тябликова. Ред. Н.Н. Боголюбов. М.: Наука, 1973. С. 411.

[4] О. И. Завьялов, В. Н. Сушко. ТМФ. 1969. Т. 1. № 2. С. 153.

[5] И. фон Нейман. Математические основы квантовой механики. М.: Наука, 1964.

[6] И. С. Градитейн, И. М. Рьжик. Таблицы интегралов, сумм, рядов и произведений. М.: Физматгиз, 1963.

[7] Н. Н. Боголюбов, Д. В. Ширков. Введение в теорию квантованных полей. М.: Наука, 1976.

[8] О. И. Завьялов. Перенормированные диаграммы Фейнмана. М.: Наука, 1979.

Поступила в редакцию 25.I.1999 г. 